Pure and Applied Mathematics Quarterly

Volume 7, Number 2

(Special Issue: In honor of

Frederick W. Gehring, Part 2 of 2$)$

383-394, 2011

\title{
The View from Above
}

\author{
Albert Marden
}

\begin{abstract}
We will consider plane regions $\Omega \subset \mathbb{C}, \Omega \neq \mathbb{C}$. This article is an exposition of the theory, initiated by Sullivan and Thurston, governing the geometric relationship of $\Omega$ to a particular surface in upper half of euclidean 3-space that will be explicitly constructed below. It lies over $\Omega$ as the dome in a domed stadium lies over the playing field. The fact of the geometric relationship opens a new direction for viewing $\Omega$ itself. It is astonishing that in the case $\Omega$ is simply connected, there is a universal relationship independent of the particular shape of $\Omega$. There are important applications to complex analysis and to the study of hyperbolic 3-manifolds.
\end{abstract}

Keywords: quasiconformal, uniformly perfect, floor-to-dome map, Sullivan Theorem

\section{UNIFORMLY PERFECT REGIONS}

We begin by addressing a topic in complex analysis that is required later. Suppose $\Omega \subset \mathbb{C}, \Omega \neq \mathbb{C}$, is simply connected. A Riemann map from the unit disk $\mathbb{D}$ carries the hyperbolic metric $d s=\frac{2|d z|}{1-|z|^{2}}$ in $\mathbb{D}$ to what is called the hyperbolic metric $d s=\rho(z)|d z|$ in $\Omega$. It is a classical result that the density $\rho(z)$ is comparable to the inverse of the shortest euclidean distance from $z$ to the boundary:

$$
\frac{1}{2 \operatorname{dist}(z, \partial \Omega)} \leq \rho(z) \leq \frac{2}{\operatorname{dist}(z, \partial \Omega)}
$$

The validity of the left inequality is a consequence of the Koebe-1/4 Theorem.

Received December 10, 2007.

This is an expanded version of a talk given at the Warwick conference honoring the 70th birthday of David Epstein. 
The obvious question is whether, perhaps for a constant $\neq 2$, the inequality also holds for multiply connected regions. However a simple counterexample is provided by the once-punctured disk, for which the density can be explicitly computed.

The answer is provided by the following statement, which consolidates results of [1] and [15] ((i) and (ii)), and of [13] ((iii) and (iv)).

Theorem 1.1. Given a multiply connected region $\Omega \subset \mathbb{C}$ with a hyperbolic metric $d s=\rho(z)|d z|$, the following statements are equivalent:

(i). There exists $0<C<\infty$ for which

$$
\frac{1}{C \operatorname{dist}(z, \partial \Omega)} \leq \rho(z) \leq \frac{C}{\operatorname{dist}(z, \partial \Omega)}
$$

(ii). There exists $0<C_{1}<\infty$ such that any annular region $A \subset \mathbb{C} \backslash \partial \Omega$ separating $\partial \Omega$ satisfies $\operatorname{Modulus}(A)<C_{1}$.

(iii). $\Omega$ has bounded geometry: There is a uniform positive lower bound for the hyperbolic injectivity radii in $\Omega$.

(iv). There does not exist a blow-up of $\Omega$ resulting in a region $\Omega^{*}$ with an isolated boundary point.

\section{REMARKS.}

(i). It is the left side that is at issue. The right side is always true, in fact with $C=2$.

(ii). The larger the modulus, the more $A$ looks like a once punctured disk.

(iii). Bounded injectivity radius means there exists $\delta>0$ such that the hyperbolic disk of radius $\delta$ about each $z \in \Omega$ is embedded. In particular there are no arbitrarily short closed geodesics.

(iv). There does not exist a sequence of Möbius transformations $\left\{T_{n}\right\}$ such that the sequence $\left\{\Omega_{n}=T_{n}(\Omega)\right\}$ converges (in the sense of Carathéodory kernel convergence) to a region $\Omega^{*}$ with an isolated boundary point.

A region $\Omega$ satisfying the properties of the Theorem is called uniformly perfect. Although this notion was really designed for multiply, especially infinitely connected, regions we will include simply connected regions $\neq \mathbb{C}$ under its umbrella. The terminology is also used for a closed set $\Lambda \subset \mathbb{C} \cup\{\infty\} \equiv \mathbb{S}^{2}$, typically with infinitely many components like a Cantor set. In our case $\Lambda=\mathbb{S}^{2} \backslash \Omega$. 
In short, the boundary of a uniformly perfect region can contain no isolated points; in fact the boundary points must be bunched together sufficiently densely to prevent separating annuli of large modulus.

The constant $C=C(\Omega)$ appearing in (i) will be called the uniformly perfect constant for $\Omega$. One could use the constant $C_{1}$ in (ii) just as well. What is important is that the constant be bounded, and $C$ is so if and only if $C_{1}$ is. We know of no formula precisely relating $C$ to $C_{1}$ - or to other constants that could be used as well.

The papers [1], [15], [14] include additional equivalent conditions. One of these leads to the following conclusion.

Theorem 1.2 ([15]). If $\Omega$ is a component of the region of discontinuity of a nonelementary, finitely generated kleinian group, then $\Omega$ is uniformly perfect.

While the limit sets of such groups have strictly positive Hausdorff dimensions, a positive Hausdorff dimension cannot in itself insure uniform perfectness.

\section{Hyperbolic CONVEX HUlls}

We will work with the upper halfspace model $\left\{(z, t): z \in \mathbb{S}^{2}, t>0\right\}$ of hyperbolic 3 -space $\mathbb{H}^{3}$. The geodesics are semicircles and vertical halflines orthogonal to $\mathbb{C}$, and the hyperbolic planes are hemispheres and vertical halfplanes also orthogonal to $C$. The hyperbolic metric is $d s=\frac{|d \vec{x}|}{t}$.

With respect to $\mathbb{H}^{3}$ we consider $\partial \mathbb{H}^{3} \equiv \mathbb{S}^{2}$ as the "sphere at $\infty$ " or the "conformal boundary". Although $\mathbb{H}^{3}$ itself is complete in its metric, a geodesic line or plane is bounded by a pair of points or a circle on $\partial \mathbb{H}^{3}$. In fact the group of orientation preserving isometries of $\mathbb{H}^{3}$ extends to $\partial \mathbb{H}^{3}=\mathbb{S}^{2}$ where it becomes the group of all orientation preserving Möbius transformations.

Let $\Lambda \subset \mathbb{S}^{2}$ be a closed set with nonempty complement $\Omega$. The hyperbolic convex hull $\mathcal{C}(\Lambda) \subset \mathbb{H}^{3}$ of $\Lambda$ is defined as follows. Set $\Omega=\mathbb{S}^{2} \backslash \Lambda$.

Consider a maximal open disk $D \subset \Omega$, a disk not contained in a larger disk in $\Omega$. Then the circle $C_{D}=\partial D \subset \bar{\Omega}$ meets $\Lambda$ in at least two points. Denote by $C_{D}^{*}$ the hemisphere - the hyperbolic plane in $\mathbb{H}^{3}$-rising from $C_{D}$. It divides upper halfspace $\mathbb{H}^{3}$ into two parts. Denote by $H\left(C_{D}^{*}\right)$ the part- the hyperbolic halfspace - that is bounded by $\mathbb{S}^{2} \backslash D$. The convex hull is defined to be

$$
\mathcal{C}(\Lambda)=\cap_{D} H\left(C_{D}^{*}\right) \subset \mathbb{H}^{3} .
$$


From now on, we will assume that $\Omega \subset \mathbb{C}$ is a connected open set and $\Lambda$ is its complement with respect to $\mathbb{S}^{2}$.

\section{EXAMPLES.}

(i.) $\Lambda=\{0\} \cup\{\infty\}$ then $\mathcal{C}(\Lambda)$ is the vertical halfline between 0 and $\infty$. It is as degenerate as the convex hull can become.

(ii.) The following example is not quite as degenerate. Let $\Lambda$ be the nonnegative real axis $\{x: 0 \leq x \leq \infty\}$. The convex hull is the vertical quarterplane rising from $\Lambda$. Again it has no interior, but it has two faces, the two sides of the wall. This example is an extreme case of the following.

(iii.) $\Lambda$ is the closed interior of the wedge $W=\{z \in \mathbb{C}: 0<\operatorname{Arg}(z)<\pi\}$. Then $\mathcal{C}(\Lambda)$ is the closed chimney rising from $W$ bounded by the quarterplanes rising from the edges of $W$.

(iv.) If instead $\Lambda$ is taken to be the closed exterior of $W$, then $\mathcal{C}(\Lambda)$ is the solid halfcone resting on $W$ whose relative boundary in $\mathbb{H}^{3}$ is the halfcone which is the envelope of the hemispheres swept out by maximal disks.

(v.) Suppose $\Lambda$ is the closed exterior of an equilateral triangle so that $\mathcal{C}(\Lambda)$ lies over the interior. Its relative boundary consists of three conical pieces rising from the three vertices which smoothly merge into the hemisphere over the inscribed circle.

In fact, as the Examples (iv), (v) illustrate, we have

Theorem 2.1 ([12]). Suppose $\Omega \subset \mathbb{C}$ is euclidean convex and $\Lambda$ is the closed exterior of $\Omega$. Then the relative boundary $\partial \mathfrak{C}(\Lambda) \subset \mathbb{H}^{3}$ lying over $\Omega$ is a $C^{1}$ surface.

2.1. The dome. We use the terminology $\operatorname{Dome}(\Omega)$ for the relative boundary $\partial \mathcal{C}(\Lambda)$ in $\mathbb{H}^{3}$; in this context, $\Omega$ is referred to as the floor.

It was Thurston who brought us the tools to analyze the dome. The dome is the union of bending lines and flat pieces. Here are some properties;

- $\partial \operatorname{Dome}(\Omega)=\partial \Omega$.

- A bending line $\ell \subset \operatorname{Dome}(\Omega)$ is a (hyperbolic) geodesic with endpoints in $\Lambda$. The bending lines form a closed set $\Lambda$ of mutually disjoint lines in $\mathbb{H}^{3}$, called the bending lamination. Two or more bending lines may share an endpoint. Possibly the dome is itself the union of bending lines. 
- A flat piece is a hyperbolic polygon lying the dome which also lies in some maximal $C_{D}^{*}$. It will have a finite or infinite number of edges, each of which is a bending line.

2.2. The bending measure. In Examples (ii) and (iii), the dome has one bending line. For these cases we see that the dome results by bending a copy of the hyperbolic plane by an angle $0<\theta<\pi$. We will measure the angle so that the extreme case $\theta=0$ corresponds to no bending at all while $\theta=\pi$ corresponds to the plane being folded over itself. This choice of $\theta$ is referred to as the exterior bending angle. When working in upper halfspace, the most convenient model of $\mathbb{H}^{2}$ to start with is the vertical halfplane rising from $\mathbb{R}$.

If $C_{1}, C_{2}$ are two intersecting circles, the exterior bending angle is the angle which lies outside one of the circles and inside the other. It is also the exterior bending angle between the two intersecting hyperbolic planes rising from the circles.

If the dome has a finite number of bending lines $\left\{\ell_{i}\right\}$ we put an atomic measure along each of the lines whose value is the corresponding exterior bending angle $\theta_{i}$. We can then introduce the corresponding transverse, nonnegative, bending measure $\mu$ : If $\sigma$ is any simple arc in the dome whose endpoints lie in flat pieces which crosses each bending line at most once, define

$$
\mu(\sigma)=\sum_{\ell_{k} \cap \sigma \neq \emptyset} \theta_{k}
$$

In general however there is an uncountable set of bending lines. In this case the transverse measure $\mu$ must be defined by a process akin to Riemann integration on transverse segments $\sigma$. The transversals can be taken as geodesic segments in the dome (see Theorem 2.2 below). Complete details are in [8]. The conclusion is that there exists a measure $\mu$ on transverse segments $\sigma$ with the properties

- The support of $\mu$ is contained in the bending lamination $\Lambda$.

- If $\sigma \cap \Lambda$ has a finite number of points, them $\mu(\sigma)=\sum \theta_{k}$ where the $\left\{\theta_{k}\right\}$ are the exterior bending angles at the crossing points.

- $\mu(\sigma) \in[0, \infty]$ is a countably additive Borel measure.

- $\mu\left(\sigma_{1}\right)=\mu\left(\sigma_{2}\right)$ if the two segments have endpoints in the same flat pieces, and are isotopic through such transversals.

2.3. The Riemann mapping theorem for domes. The following theorem is due to Thurston. A complete proof appears in [8]. 


\section{Theorem 2.2.}

- The ambient hyperbolic metric in $\mathbb{H}^{3}$ restricts to a path metric on $\operatorname{Dome}(\Omega)$, called its hyperbolic metric.

- (Riemann Mapping Theorem) If $\Omega$ is simply connected, there is an isometry ("unbending")

$$
\iota: \operatorname{Dome}(\Omega) \rightarrow \mathbb{D}=\{z \in \mathbb{C}:|z|<1\} .
$$

- (Uniformization theorem) If $\Omega$ is not simply connected, there is a local isometry from the universal cover:

$$
\pi_{\text {Dome }(\Omega)}: \mathbb{D} \rightarrow \operatorname{Dome}(\Omega) .
$$

The image of the bending lines becomes a geodesic lamination $\Lambda$ in $\mathbb{D}$. The bending measure becomes a transverse measure $\mu$ in $\mathbb{D} ;(\Lambda, \mu) \subset \mathbb{D}$ is a measured lamination.

Conversely, given a geodesic lamination $\Lambda \subset \mathbb{D}$, and a transverse measure $\mu$, by a process of successively bending $\mathbb{D}$ there results a "pleated surface" in $\mathbb{H}^{3}$, uniquely determined up to isometry. In general, it is unlikely to be embedded and may even be dense in $\mathbb{H}^{3}$. However the following facts are known.

Introduce a norm on bending measures by:

$$
\|\mu\|=\sup _{\operatorname{Len}(\sigma) \leq 1} \mu(\sigma) .
$$

In typical cases under consideration the norm is or is assumed to be finite.

\section{Theorem 2.3.}

- Suppose $\mu$ is the bending measure for the dome over a simply connected region. Then $\|\mu\|<4.88[5]$.

- In the opposite direction, there exists a constant $0<C \leq 2 \arcsin (\tanh (1 / 2)) \sim$ .96 such that if $(\Lambda, \mu)$ is a measured lamination in $\mathbb{D}$ that satisfies $\|\mu\| \leq$ $C$, then $(\Lambda, \mu)$ comes from the bending measure of the dome over a quasidisk [10].

It is conjectured that $C$ is equal to the indicated numerical upper bound.

\section{The Nearest Point Retraction}

The basic tool in relating the dome to the floor is the nearest point retraction. It is defined as follows. For $z \in \Omega$, the family of horospheres in $\mathbb{H}^{3}$ at $z$ consists of the family of euclidean spheres in upper halfspace which are tangent to $\mathbb{C}$ at $z$. 
If $z=\infty$ they form the family of horizontal euclidean planes in upper halfspace. (Horospheres are not geodesic spheres in the hyperbolic metric.) At each $z \in \Omega$ there is a smallest horosphere that meets $\operatorname{Dome}(\Omega)$, necessarily at a single point $r(z)$.

The map $r: z \mapsto r(z) \in \operatorname{Dome}(\Omega)$ is continuous and surjective but not necessarily a local homeomorphism. It has a continuous extension to the common boundary $\partial \Omega=\partial \operatorname{Dome}(\Omega)$ where it is the identity. (There are some subtleties here - see [11].)

If $r(z)$ is in (the interior of) a flat piece $f$, then the geodesic ray from $z$ to $r(z)$ is orthogonal to $f$ at $r(z)$. In fact $r$ is a conformal map of $r^{-1}(f) \in \mathbb{S}^{2}$ onto $f$.

The failure of $r$ to be a homeomorphism occurs in the presence of an isolated bending line $\ell \subset \operatorname{Dome}(\Omega)$. In this case $r^{-1}(\ell) \subset \Omega$ is a crescent: the intersection of two maximal disks in $\Omega$. Its interior vertex angles are $\pi-\theta$ where $\theta$ is the exterior bending angle along the bending line. The crescent is bounded by an arc of each maximal circle. It is foliated by the circular arcs which are preimages of the points along $\ell$.

Thus if there are no isolated bending lines, then $r: \Omega \rightarrow \operatorname{Dome}(\Omega)$ is a homeomorphism.

One might ask, does $r$ have stronger continuity properties? For example, is $r$ Lipschitz: Does there exist $L>0$ such that in the respective hyperbolic metrics, and for all $z, w \in \Omega$,

$$
d_{\text {Dome }(\Omega)}(r(z), r(w)) \leq L d_{\Omega}(z, w) ?
$$

If $\Omega$ is simply connected the answer is given by

Theorem $3.1([10])$. When $\Omega \neq \mathbb{C}$ is simply connected, $r: \Omega \rightarrow \operatorname{Dome}(\Omega)$ is 2-Lipschitz. The constant is sharp.

On the other hand if $\Omega$ is not uniformally perfect, $r$ cannot be Lipschitz.

Conversely, in many cases of uniformly perfect regions, it is known ([6], [10]) that $r$ is Lipschitz.

It is conjectured [13] that $r$ is Lipschitz if and only if $\Omega$ is uniformly perfect, with the Lipschitz constant depending only on the uniformly perfect constant.

\subsection{The fundamental theorem for nearest point retractions.}


Theorem 3.2 ([13]). Necessary and sufficient for

$$
r: \Omega \rightarrow \operatorname{Dome}(\Omega)
$$

to be a quasiisometry is that $\Omega$ be uniformly perfect.

Specifically, there exists $L \geq 1$, depending only on the uniformly perfect constant for $\Omega$, such that for all $z, w \in \Omega$ and the respective hyperbolic metrics,

$$
\frac{d_{\Omega}(z, w)}{L}-1<d_{\operatorname{Dome}(\Omega)}(r(z), r(w))<L d_{\Omega}(z, w)+1 .
$$

In short, $r$ is referred to as being "long range bilipschitz".

There is a lot of topological flexibility in constructing quasiisometries as they do not need to be continuous. Quasiisometries are of fundamental importance in hyperbolic geometry because of the following fact. A quasiisometry of the unit disk $F: \mathbb{D} \rightarrow \mathbb{D}$ extends to become a homeomorphism $F^{*}: \partial \mathbb{D} \rightarrow \partial \mathbb{D}$. Moreover the extension has the much stronger property of quasisymmetry (1-quasiconformal). The class of quasisymmetric mappings of $\partial \mathbb{D}$ is in turn precisely the class of homeomorphisms that have quasiconformal extensions to $\mathbb{D}$. A corresponding property holds for all real hyperbolic spaces.

\section{TeichmüLler Distance Between REgions AND their DOMES}

The goal of this note is the present the following rather amazing fact discovered in the simply connected case by Sullivan and Thurston, and first recorded by Sullivan [16]. The first complete proof for the simply connected case appears in [8] with an alternate, much shorter proof in [11]. The shortest proof is the one presented here, which strengthens the original theorem while equally covering the multiply connected case.

Theorem 4.1 ([16], [8], [11], [13]). Given $0<C^{*}<\infty$ there exists $1<K^{*}=$ $K^{*}\left(C^{*}\right)<\infty$ with the following property. Corresponding to any $\Omega \subset \mathbb{C}$ with uniformly perfect constant $C(\Omega) \leq C^{*}$, there exists a $K$-quasiconformal, bilipschitz mapping $\Psi$ with $K \leq K^{*}$,

$$
\Psi: \Omega \longrightarrow \operatorname{Dome}(\Omega)
$$

that satisfies

(i) $\Psi$ has a continuous extension to $\partial \Omega=\partial \operatorname{Dome}(\Omega)$ that fixes every point.

(ii) For some $M=M\left(C^{*}\right)<\infty$ and all $z \in \Omega$,

$$
d_{\text {Dome }(\Omega)}(\Psi(z), r(z)) \leq M .
$$


(iii) $\lim _{C^{*} \rightarrow \infty} K^{*}\left(C^{*}\right)=\infty$.

REMARKS.

- If $\Omega$ is not uniformly perfect, (i) can hold while (ii) does not. An example is the once punctured disk.

- If $\Omega$ is simply connected, $C^{*}=2$ is independent of $\Omega$.

Proof outline. Denote a Riemann mapping or projection from the universal covering surface as the case may be by $\pi_{\Omega}$ or $\pi_{\text {Dome }(\Omega)}$ respectively.

$F=\pi_{\text {Dome }(\Omega)}^{-1} \circ r \circ \pi_{\Omega}: \mathbb{D} \rightarrow \mathbb{D}$ is $(L, 1)$-quasiisometric in the hyperbolic metric on $\mathbb{D}$. It is equivariant in that it induces an isomorphism between the two groups of deck transformations.

$\Longrightarrow F$ extends to an equivariant quasisymmetry $F^{*}: \partial \mathbb{D} \rightarrow \partial \mathbb{D}$.

$\Longrightarrow$ There exists a quasiconformal, bilipschitz, equivariant extension of $F^{*}$ back to $\mathbb{D}$. The Douady-Earle extension $E: \mathbb{D} \rightarrow \mathbb{D}[7]$ is made to order for this purpose.

$\Longrightarrow$ Since $F$ and necessarily also $E$ are quasiisometries with the same boundary values, in the hyperbolic metric on $\mathbb{D}$,

$$
d_{\mathbb{D}}(E(z), F(z))<M<\infty,
$$

for some $M$ and all $z \in \mathbb{D}$.

$\Longrightarrow$ The quasiconformal, bilipschitz map

$$
\Psi=\pi_{\operatorname{Dome}(\Omega)} \circ E \circ \pi_{\Omega}^{-1}: \Omega \rightarrow \operatorname{Dome}(\Omega)
$$

is just what we are looking for.

4.1. Equivariance. The original applications of Theorem (4.1) were to the proof of the hyperbolization theorem for 3-manifolds fiber over the circle (see [17]). For these cases, $\Omega$ is a simply connected region of discontinuity for a nonelementary kleinian group $\Gamma$. For the application we need to know that the function $\Psi$ constructed in Theorem (4.1) is equivariant under $\Gamma$.

First of all the nearest point retraction is so equivariant. The construction of $\Psi$ is then equivariant as well.

We will not go further into a discussion of applications to hyperbolic 3-manifolds. 


\section{Numerical estimates FOR $K$}

In the case of simply connected regions it is natural to ask, what exactly is the optimal number $K^{*}$ whose existence is demonstrated in Theorem (4.1)? It was suggested by Thurston in the equivariant context [17] that $K^{*}=2$. That this may be true is suggested by the simple Example (ii) of the convex hull of the nonnegative real axis. In this case $K^{*}$ can be computed explicitly and it is exactly two. Could this represent the extreme case?

The problem can be separated into two parts.

- Equivariant conjecture. $K^{*}=2$ for all $\Psi: \Omega \rightarrow \operatorname{Dome}(\Omega)$ which are equivariant if $\Omega$ is the region of discontinuity of a nonelementary kleinian group.

- Nonequivariant conjecture. $K^{*}=2$ without any requirement that the map $\Psi$ be equivariant.

The state of current knowledge is as follows. The theorems presented below are actually amalgams of hard-to-prove individual theorems due to the mathematicians indicated.

Theorem 5.1. For all simply connected regions $\Omega \subset \mathbb{C}, \Omega \neq \mathbb{C}$ the following estimates for $K^{*}$ hold:

- $([8], 1986$.$) For both equivariant and nonequivariant cases, K^{*} \leq 82.8$.

- ([11], 2006.) For both equivariant and nonequivariant cases, $K^{*} \leq 13.88$.

- $\left([3], 2002\right.$.) For the nonequivariant case, $K^{*} \leq 7.82$.

Theorem 5.2. The universal constant $K^{*}$ for simply connected regions $\{\Omega\}$ satisfies the following estimates,

- Equivariant counterexample. ([10], 2004.) For the equivariant case, $K^{*}$ is greater than two. This is true in particular for certain regions close to the boundary of the once-punctured torus quasifuchsian space.

- Nonequivariant counterexample. ([9], 2005.) When equivariance is not required, $K^{*}$ is also greater than two-specifically, for a certain logarithmic spiral, $K^{*}$ must be greater than 2.1 .

Thus the value of the best $K^{*}$ and the extremal regions remain mysterious. Its discovery may have application to analysis on plane regions $\Omega$ especially those with bad boundaries, see [2], [4].

In the opposite direction we have 
Theorem 5.3 ([12], 2006.). For euclidean convex regions $\{\Omega\}, K^{*} \leq 2$.

\section{REFERENCES}

[1] A. Beardon and Ch. Pommerenke, "The Poincaré metric of plane domains," J. London Math. Soc. 18 (1978), 475-483.

[2] C. Bishop, "Quasiconformal Lipschitz maps, Sullivan's convex hull theorem, and Brennan's conjecture," Arch. Math. 40 (2002), 1-26.

[3] C. Bishop, "An explicit constant for Sullivan's convex hull theorem," in Proc. of 2002 AhlforsBers Colloq. Contemp. Math. bf 355, Amer. Math. Soc. 2004.

[4] C. Bishop, "A fast approximation to the Riemann map," preprint available at www.math.sunysb.edu/ ${ }^{\sim}$ bishop.

[5] M. Bridgeman and R.D. Canary, "From the boundary of the convex core to the conformal boundary," Geom. Dedicata 96 (2003), 211-240.

[6] R.D. Canary, "The conformal boundary and the boundary of the convex core," Duke Math. J. 106 (2001), 193-207.

[7] A. Douady and C.J. Earle, "Conformally natural extension of homeomorphisms of the circle," Acta Math. 157 (1986), 23-48.

[8] D.B.A. Epstein and A. Marden, "Convex hulls in hyperbolic space, a theorem of Sullivan, and measured pleated surfaces," in Fundamentals of Hyperbolic Geometry: Selected Expositions, R.D. Canary, D.B.A. Epstein, A. Marden, eds., London Math. Soc. Lecture Notes 328, 2005 (reprint with addendum of an article in LMS Lecture Notes 111).

[9] D.B.A. Epstein and V. Markovic, "The logarithmic spiral: a counterexample to the $K=2$ conjecture," Annals of Math. 161 (2006), 925-957.

[10] D.B.A. Epstein, V. Markovic, and A. Marden, "Quasiconformal homeomorphisms and the convex hull boundary," Annals of Math. 159 (2004), 305-336.

[11] D.B.A. Epstein, A. Marden, and V. Markovic, "Complex earthquakes and deformations of the unit disk," Jour. Diff. Geom. 73 (2006), 119-166.

[12] D.B.A. Epstein, A. Marden, and V. Markovic, "Convex regions in the plane and their domes," Proc. London Math. Soc. 92 (2006), 624-654.

[13] A. Marden and V. Markovic, "Characterization of plane regions that support quasiconformal mappings to their domes," Bull. London Math. Soc. 39 (2007), 962-972.

[14] Ch. Pommerenke, "Uniformly perfect sets and the Poincaré metric," Ark. Math. 32 (1979), 192-199.

[15] Ch. Pommerenke, "Uniformly perfect sets and fuchsian groups," Analysis 4 (1984), 299321 .

[16] D. Sullivan, "Travaux de Thurston sur les groupes quasi-fuchsiens et les variétés hyperboliques de dimension 3 fibrés sur $\mathbb{S}^{1}$," in LNM 842, Springer-Verlag, 1981.

[17] W.P. Thurston, "Hyperbolic structure on 3-manifolds, II: Surface groups and 3-manifolds which fiber over the circle," available at http://arXiv.org —arXiv:math.GT/9801039.

Albert Marden

School of Mathematics

University of Minnesota 
Minneapolis, MN 55455

E-mail: am@umn.edu 